\title{
ON THE ZEROS OF SOLUTIONS OF ORDINARY DIFFERENTIAL EQUATIONS OF THE SECOND ORDER
}

\author{
A. S. GALBRAITH
}

This note gives a proof of the following theorem:

In the differential equation

$$
y^{\prime \prime}+p(x) y=0,
$$

where primes mean derivatives, suppose $p(x)$ to be positive or zero, monotonic and concave (no point of an arc lies below its chord) in some closed interval $[a, b]$. If

$$
\int_{a}^{b} p(x) d x \geqq(9 / 8) n^{2} \pi^{2} /(b-a),
$$

where $n$ is an integer, then every solution of (1) has at least $n$ zeros in $[a, b]$. The number $9 / 8$ cannot be replaced by a smaller one.

A theorem similar to this, but with more restrictive hypotheses, has been proved by Makai [5].

Related theorems have been proved by a number of authors, back as far as Liouville. More recent examples are given in the references.

The proof depends on three lemmas.

LEMMA 1. If the equation

$$
y^{\prime \prime}+q(x) y=0,
$$

where $q(x)$ is continuous, has a solution with consecutive zeros at $x=c$ and $x=d$, and if

$$
\int_{0}^{d} q(x) \cos (2 \pi(x-c) /(d-c)) d x \leqq 0,
$$

then

$$
\int_{c}^{d} q(x) d x \leqq \pi^{2} /(d-c) .
$$

Proof. Let $y(x)$ be the solution referred to, and let $z(x)=(2 /(d-c))^{1 / 2} \sin (\pi(x-c) /(d-c))$, so that $\int_{0}^{d} z^{2} d x=1$.

Now

Received by the editors May 3, 1965. 


$$
\begin{aligned}
0 & \leqq \int_{c}^{d}\left(\left(z^{\prime} y-z y^{\prime}\right)^{2} / y^{2}\right) d x=\int_{c}^{d}\left(z^{\prime} y-z y^{\prime}\right)(z / y)^{\prime} d x \\
& =\left.\left(z^{\prime} y-z y^{\prime}\right)(z / y)\right|_{c} ^{d}-\int_{c}^{d}(z / y)\left(z^{\prime \prime} y-z y^{\prime \prime}\right) d x \\
& =\int_{c}^{d}\left(\pi^{2} /(d-c)^{2}-q(x)\right) z^{2} d x,
\end{aligned}
$$

from (3). Since $y(x)$ and $z(x)$ have simple zeros at $x=c$ and $x=d$, their ratio has a limit at each end of the interval, so the integrated term vanishes. Then

$$
\begin{aligned}
\pi^{2} /(d-c)^{2} & \geqq \int_{c}^{d} q(x) z^{2} d x \\
& =(1 /(d-c)) \int_{c}^{d} q(x)[1-\cos (2 \pi(x-c) /(d-c))] d x
\end{aligned}
$$

From this and (4), (5) follows.

COROLlARy. The number $s$ is not less than $d$, if $s$ is determined by

$$
\int_{c}^{s} q(x) d x=\pi^{2} /(s-c) .
$$

LEMMA 2. If $p(x)$ is concave, then

$$
\int_{c}^{d} p(x) \cos (2 \pi(x-c) /(d-c)) d x \leqq 0 .
$$

This lemma is due to E. Makai [4], pp. 370-371.

Lemma 3. Let $\left\{x_{i}\right\}$ and $\left\{x_{i}^{\prime}\right\}, i=0,1,2, \cdots, n$, be two sets of numbers such that

(a) $x_{0}<x_{1}<x_{2}<\cdots<x_{n}, x_{0}^{\prime}<x_{1}^{\prime}<x_{2}^{\prime}<\cdots<x_{n}^{\prime}$;

(b) $x_{1}-x_{0} \geqq x_{2}-x_{1} \geqq x_{3}-x_{2} \geqq \cdots x_{n}-x_{n-1}$,

and similarly for the $\left\{x_{i}^{\prime}\right\}$;

(c) $x_{i}^{\prime} \leqq x_{i}, i=1,2, \cdots, n-1$;

(d) $x_{0}=x_{0}^{\prime}, x_{n}=x_{n}^{\prime}$.

Then

$$
\sum_{i=1}^{n} 1 /\left(x_{i}^{\prime}-x_{i-1}^{\prime}\right) \leqq \sum_{i=1}^{n} 1 /\left(x_{i}-x_{i-1}\right)
$$

Proof. The case $n=1$ is trivial, and if $n=2$ the proof is elementary. 
To complete the proof by induction, let $S_{n}^{\prime}$ and $S_{n}$ stand for the left and right members of (6) respectively. Then $S_{n}^{\prime} \leqq S_{n}$ must be shown to imply $S_{n+1}^{\prime} \leqq S_{n+1}$, with $n$ replaced by $n+1$ in (a), (b), (c) and (d). Let $g$ be the least of $x_{i}-x_{i}^{\prime}$, and let $x_{i}^{\prime \prime}=x_{i}^{\prime}+g, i=1,2, \cdots, n$, so that $x_{i}^{\prime \prime}-x_{i-1}^{\prime \prime}=x_{i}^{\prime}-x_{i-1}^{\prime}$. Let $S_{n+1}^{\prime \prime}$ be formed from $S_{n+1}^{\prime}$ by substituting $x_{i}^{\prime \prime}$ for $x_{i}^{\prime} .\left(x_{0}^{\prime \prime}=x_{0}^{\prime}=x_{0}, x_{n+1}^{\prime \prime}=x_{n+1}^{\prime}=x_{n+1}\right.$. $)$ Then

$$
\begin{aligned}
S_{n+1}^{\prime \prime} & -S_{n+1}^{\prime} \\
& =1 /\left(x_{n+1}-x_{n}^{\prime \prime}\right)-1 /\left(x_{n+1}-x_{n}^{\prime}\right)+1 /\left(x_{1}^{\prime \prime}-x_{0}\right)-1 /\left(x_{1}^{\prime}-x_{0}\right) \\
& =g\left[1 /\left(x_{n+1}-x_{n}^{\prime \prime}\right)\left(x_{n+1}-x_{n}^{\prime}\right)-1 /\left(x_{1}^{\prime \prime}-x_{0}\right)\left(x_{1}^{\prime}-x_{0}\right)\right] .
\end{aligned}
$$

Now $x_{n+1}-x_{n}^{\prime \prime}=x_{n+1}-x_{n}+\left(x_{n}-x_{n}^{\prime}\right)-g \geqq x_{n+1}-x_{n}>0$, and $x_{n+1}-x_{n}^{\prime \prime}$ $\leqq x_{n+1}-x_{n}^{\prime} \leqq x_{1}^{\prime}-x_{0}$ by (c) above; while $x_{1}^{\prime \prime}-x_{0} \geqq x_{1}^{\prime}-x_{0}$. Hence the first denominator in the square bracket is not greater than the second, so that the bracket is positive or zero. Then $S_{n+1}^{\prime \prime}-S_{n+1}^{\prime} \geqq 0$.

But for at least one value of $i$, say $i=k, 0<k<n+1, x_{k}^{\prime \prime}=x_{k}$. Then $S_{n+1}$ and $S_{n+1}^{\prime \prime}$ can each be broken into two sums, from $i=1$ to $i=k$ and from $i=k+1$ to $i=n+1$ respectively. Each of these latter sums contains no more than $n$ terms and satisfies the hypotheses of the lemma. Hence the induction hypothesis applies to each, and their addition yields $S_{n+1}-S_{n+1}^{\prime \prime} \geqq 0$. Addition of the previous inequality gives the lemma. (This proof, by E. Makai, Jr., was kindly sent to the author by the referee.)

Proof of THE THEOREM. Consider first that $p(x)$ has the special form

$$
r(x)=2 A n^{2} \pi^{2} x,
$$

where $A$ is a positive constant and $[a, b]$ is $[0,1]$. Inequality (4) is satisfied, and it will appear later that $A$ can be $9 / 8$. Choose a solution $y(x)$ with $y(0)=0$. Successive applications of Lemma 1 show that the succeeding zeros of $y(x)$ precede respectively the numbers $x_{1}, x_{2}, \cdots$, where the $x_{i}$ are determined by the equations

$$
\begin{aligned}
& \int_{0}^{x_{1}} r(x) d x=\pi^{2} / x_{1} \text { or } \operatorname{An}^{2} x_{1}^{3}=1 \\
& \int_{x_{1}}^{x_{2}} r(x) d x=\pi^{2} /\left(x_{2}-x_{1}\right) \text { or } \operatorname{An}^{2}\left(x_{2}^{2}-x_{1}^{2}\right)\left(x_{2}-x_{1}\right)=1 \text {, etc. }
\end{aligned}
$$

For $n=1$, the theorem is true by Lemma 1. Assume it true for $n$. For $n+1$, the points $X_{i}$ corresponding to the $x_{i}$ are $X_{i}=(n /(n+1))^{2 / 3} x_{i}$, as is seen by substitution. The theorem will be true for $n+1$ if

$$
A(n+1)^{2}\left(X_{n+1}^{2}-X_{n}^{2}\right)\left(X_{n+1}-X_{n}\right)=1
$$


while $X_{n+1} \leqq 1$, or if

$$
A(n+1)^{2}\left\{1-(n /(n+1))^{4 / 3} x_{n}^{2}\right\}\left\{1-(n /(n+1))^{2 / 3} x_{n}\right\} \geqq 1 .
$$

Since $x_{n} \leqq 1, A$ can be chosen so that

$$
A(n+1)^{2}\left\{1-(n /(n+1))^{4 / 3}\right\}\left\{1-(n /(n+1))^{2 / 3}\right\} \geqq 1,
$$

or

$$
A\left\{(n+1)^{2 / 3}-n^{2 / 3}\right\} 2\left\{(n+1)^{2 / 3}+n^{2 / 3}\right\} \geqq 1 .
$$

Let the part in braces be $f(n)$. Then $f(1)>0.8914$, since $2^{2 / 3}>1.587$. Then if $A=9 / 8, A f(1)>1$.

The behavior of $f(n)$ for large values of $n$ can be examined by treating $n$ as a continuous variable, increasing without limit. It can be shown by elementary arguments that $f^{\prime} / f$ is negative and that the limit of $f(n)$ as $n$ increases is $8 / 9$. Hence if $A=9 / 8$ the theorem is true for the function $r(x)$ considered.

Now let $p(x)$ be a function different from $r(x)$, and satisfying the hypotheses of the theorem. Since $\int_{0}^{1} p(x) d x \geqq(9 / 8) n^{2} \pi^{2}, p(x)$ must be greater than $r(x)$ near $x=0$ and less (perhaps) near $x=1$. Suppose first that $p(x)$ is increasing. The equations $(7)$, with $p(x)$ for $r(x)$, will determine numbers $x_{1}^{\prime}, x_{2}^{\prime}, \cdots$, which are not less than the respective zeros, by Lemma 1 , and such that $x_{1}^{\prime} \geqq x_{2}^{\prime}-x_{1}^{\prime} \geqq \cdots$.

Now $x_{i}^{\prime} \leqq x_{i}, i=1,2, \cdots, n$. For if not, let $x_{i}^{\prime} \leqq x_{i}, i=1,2, \cdots$, $j-1$, and suppose $x_{j}^{\prime}>x_{j}$. Then

$$
\begin{aligned}
\left(1 / \pi^{2}\right) \int_{0}^{x_{j^{\prime}}} p(x) d x & =1 / x_{1}^{\prime}+1 /\left(x_{2}^{\prime}-x_{1}^{\prime}\right)+\cdots+1 /\left(x_{j}^{\prime}-x_{j-1}^{\prime}\right) \\
& <1 / x_{1}^{\prime}+1 /\left(x_{2}^{\prime}-x_{1}^{\prime}\right)+\cdots+1 /\left(x_{j}-x_{j-1}^{\prime}\right) \\
& \leqq 1 / x_{1}+1 /\left(x_{2}-x_{1}\right)+\cdots+1 /\left(x_{j}-x_{j-1}\right)
\end{aligned}
$$

by Lemma 3 , and this last sum is $\left(1 / \pi^{2}\right) \int_{0}^{x_{j}} r(x) d x$. But if $F(x)=$ $\int_{0}^{x}(p(t)-r(t)) d t, F^{\prime}(x)=p(x)-r(x)$, which is zero at no more than one point between 0 and 1 , by the concavity of $p(x)$. Hence $F(x)$ has at most one maximum, and is positive between 0 and 1 . Since $p(x)$ is positive, $\int_{0}^{x_{j}^{\prime \prime}} p(x) d x<\int_{0}^{x^{\prime}} p(x) d x<\int_{0}^{x_{j}{ }^{\prime \prime}} r(x) d x$ by (8), so $F\left(x_{j}\right)<0$, a contradiction. Hence $x_{j}^{\prime} \leqq x_{j}, j=1,2, \cdots, n$. The inequality above will apply directly if $n=1$. Sturm's separation theorem shows that every other solution will have at least $n$ zeros. A linear change of variable from $[0,1]$ to $[a, b]$ does not affect the argument. If $p(x)$ is decreasing, the same proof can be used from right to left.

That $9 / 8$ is the best possible constant can be shown thus: The 
solution of $y^{\prime \prime}+x y=0$ that vanishes at the origin is $y(x)$ $=(x)^{1 / 2} J_{1 / 3}\left(2 x^{3 / 2} / 3\right)$, where $J()$ is the Bessel's function of the first kind. If $t=2 x^{3 / 2} / 3$, the function $Y(t)=(t)^{1 / 2} J_{1 / 3}(t)$ has zeros at points corresponding to those of $y(x)$, and satisfies the equation

$$
d^{2} Y / d t^{2}+\left(1+5 /\left(36 t^{2}\right)\right) Y=0 .
$$

The zeros $t_{n}$ of $Y(t)$ after the first will have the form

$$
t_{n}=n \pi+h+o(1 / n),
$$

where $h$ is some constant, since the interval between successive zeros approaches $\pi$ as $n$ becomes infinite. If a constant $B<9 / 8$ could be used in (2), that inequality would show that the number $v_{n}$, determined by $\int_{0}^{v_{n}} x d x=B n^{2} \pi^{2} / v_{n}$, was not less than $x_{n}$, the $n$th positive zero of $J_{1 / 3}\left(2 x^{3 / 2} / 3\right)$. This would imply

$$
\begin{aligned}
B & =v_{n}^{3} / 2 n^{2} \pi^{2} \geqq x_{n}^{3} / 2 n^{2} \pi^{2}=\left(3 t_{n} / 2\right)^{2} / 2 n^{2} \pi^{2} \\
& =\left(9 / 8 n^{2} \pi^{2}\right)(n \pi+h+o(1 / n))^{2},
\end{aligned}
$$

which approaches $9 / 8$. This completes the proof.

The author is grateful to the referee for the proof of Lemma 3, for [4], and for pointing out some places where the argument was not clear.

\section{REFERENCES}

1. D. O. Banks, Upper bounds for the eigenvalues of some vibrating systems, Pacific J. Math. 11 (1961), 1183-1203.

2. Philip Hartman, Ordinary differential equations, Chapter 11, Wiley, New York, 1964.

3. Walter Leighton, On the zeros of solutions of a second order linear differential equation, J. Math. Pures Appl. 44 (1965), 297-310.

4. E. Makai, Ueber eine Eigenwertabschätzung bei gewissen homogenen linearen Differentialgleichungen zweiter Ordnung, Compositio Math. 6 (1938-1939), 368-374.

5. - Ueber die Nullstellen von Funktionen, die Lösungen Sturm-Liouville'scher Differentialgleichungen sind, Comment. Math. Helv. 16 (1944), 153-199.

6. W. E. Milne, On the degree of convergence of expansions in an infinite interval, Trans. Amer. Math. Soc. 31 (1929), 906-918.

7. Z. Nehari, Some eigenvalue estimates, J. Analyse Math. 7 (1959), 79-88.

Army Research Office, Durham 